\title{
BMJ open Management of patient adherence to medications: protocol for an online survey of doctors, pharmacists and nurses in Europe
}

\author{
Wendy Clyne, ${ }^{1}$ Comfort Mshelia, ${ }^{1}$ Stephanie Hall, ${ }^{2}$ Sarah McLachlan, ${ }^{1}$ \\ Peter Jones, ${ }^{3}$ Fabienne Dobbels, ${ }^{4}$ Todd Ruppar, ${ }^{4,5}$ Kaat Siebens, ${ }^{4}$ Val Morrison, ${ }^{6}$ \\ Sabina De Geest, ${ }^{4}$ Premyzlaw Kardas ${ }^{7}$
}

To cite: Clyne W, Mshelia C, Hall S, et al. Management of patient adherence to medications: protocol for an online survey of doctors, pharmacists and nurses in Europe. BMJ Open 2011;1: e000355. doi:10.1136/ bmjopen-2011-000355

- Prepublication history and an additional table for this paper are available online. To view these files please visit the journal online (http:// bmjopen.bmj.com).

Received 26 August 2011 Accepted 15 September 2011

This final article is available for use under the terms of the Creative Commons Attribution Non-Commercial 2.0 Licence; see http://bmjopen.bmj.com
For numbered affiliations see end of article.

Correspondence to Dr Wendy Clyne; w.clyne@mema.keele.ac.uk

\section{ABSTRACT}

Introduction: It is widely recognised that many patients do not take prescribed medicines as advised. Research in this field has commonly focused on the role of the patient in non-adherence; however, healthcare professionals can also have a major influence on patient behaviour in taking medicines. This study examines the perceptions, beliefs and behaviours of healthcare professionals-doctors, pharmacists and nurses-about patient medication adherence.

Methods and analysis: This paper describes the study protocol and online questionnaire used in a cross-sectional survey of healthcare professionals in Europe. The participating countries include Austria, Belgium, France, Greece, The Netherlands, Germany, Poland, Portugal, Switzerland, Hungary, Italy and England. The study population comprises primary care and community-based doctors, pharmacists and nurses involved in the care of adult patients taking prescribed medicines for chronic and acute illnesses. Discussion: Knowledge of the nature, extent and variability of the practices of healthcare professionals to support medication adherence could inform future service design, healthcare professional education, policy and research.

\section{ARTICLE SUMMARY}

Article focus

- A protocol for a cross-sectional survey of healthcare professionals in Europe to examine the perceptions, beliefs and behaviours of healthcare professionals-doctors, pharmacists and nurses-about patient medication adherence.

n The questionnaire used in the survey of healthcare professionals is described in detail.

Key messages

- There is an acute need for evidence regarding healthcare professionals' beliefs, perceptions and behaviour with regard to patient non-adherence to medicines.

- This protocol describes a study to address this need.

- The results of this study could guide healthcare professionals as they support patients with taking medicine in their day-to-day clinical practice.

Strengths and limitations of this study

n The survey is the largest cross-national survey of healthcare professionals' approach to medication adherence.

- Reliance on self-report data may raise concerns regarding the validity of the findings.

\section{INTRODUCTION}

Chronic diseases are a major source of disability and death worldwide. ${ }^{1}$ One way of managing chronic diseases is by taking medicines. In order for these medicines to work in the way they are intended, they need to be taken in the way they were prescribed. Taking prescribed medicines irregularly or not at all is often referred to as non-adherence. ${ }^{2}$ It is widely recognised that many patients do not take prescribed medication as advised, and the $\mathrm{WHO}^{2}$ reports that only around $50 \%$ of the general population in developed countries are adherent to treatment for chronic diseases. Poor adherence can have a negative impact on both the potential clinical benefits of treatment ${ }^{3}$ and the cost-effectiveness of medicines. ${ }^{4}{ }^{5}$ From the patient's point of view, non-adherence can also have positive consequences. For example, patients who fail to take their medicines as prescribed avoid the potential unpleasant side effects of their medicines. Patients may also benefit from a perception of autonomy and personal agency through non-adherence. Healthcare professionals have a role to play in providing support to 
patients in order to ensure that if the patient agrees to take the medicine, it is taken in a way that will maximise its benefit.

Several studies ${ }^{6}$ report on the use and effectiveness of various interventions to improve patient adherence to medicines. The effectiveness of adherence interventions, however, needs to be looked at in a broader context which includes the role of healthcare professionals. Improving the ability of healthcare professionals to properly assess the risk of non-adherence and deliver interventions aimed at reducing non-adherence may lead to more effective support offered to patients taking prescribed medicines. In the past, the focus of research in the field of adherence has been largely on the patient's role. In order to gain a fuller understanding of the problem and address the gap in current knowledge, this study, which is taking place in several countries across Europe, looks at the perceptions, beliefs and behaviours of healthcare professionals-doctors, nurses and pharmacists - with regards to patient medication adherence. Knowledge of the nature, extent and variability of the practices of healthcare professionals to support medication adherence could inform future service design, healthcare professional education, policy and research.

This study is part of a larger project on patient medication adherence funded by the European Commission called the 'ABC (Ascertaining Barriers for Compliance) Project' (http://www.abcproject.eu). The overall goal of the $\mathrm{ABC}$ project is to produce evidence-based policy recommendations for improving patient adherence and by so doing to promote safer, more effective and costeffective medicines use in Europe.

\section{METHODS AND ANALYSIS}

Design

This paper describes the protocol and questionnaire used in a cross-sectional survey of healthcare professionals in Europe. A quantitative self-report questionnaire has been designed specifically for this study. The questionnaire is administered online using the survey tool provided by SurveyMonkey. SurveyMonkey has been successfully used in published research involving surveys of health care professionals, and its use has been described in detail by Dobrow and colleagues. ${ }^{7}$ For a survey such as this with widespread geographical coverage, use of the internet should aid the logistics of survey administration.

\section{Aim}

The aim of this study is to ascertain how healthcare professionals-doctors, pharmacists and nurses-across Europe assess and support patient adherence to prescribed medication for long-term conditions, with secondary aims of assessing national and professional variations in adherence support behaviour.

\section{Objectives}

The objectives of this study are to examine healthcare professionals' perceptions of the extent of non-adherence, to determine healthcare professionals' beliefs about adherence and non-adherence as well as the behaviours of healthcare professionals to support patients with taking medicines and their perceptions about the effectiveness of those actions. The study will also consider perceived barriers to supporting adherence as well as training received for managing non-adherence as reported by healthcare professionals.

\section{Study population}

The study is being conducted in several countries across Europe including Austria, Belgium, France, Greece, The Netherlands, Germany, Poland, Portugal, Switzerland, Hungary, Italy and England. The survey is focused on primary care and community-based doctors, pharmacists and nurses. These professional groups have been selected because of their involvement in the care of adults who are prescribed medicines for chronic and acute conditions.

\section{Inclusion criteria}

Healthcare professionals who satisfy the following criteria are eligible for inclusion in the survey:

a. they are currently employed as medical doctors, nurses or pharmacists;

b. they work mainly with adults;

c. they work mainly in the community or primary care;

d. they work either in a private or public healthcare system (or both);

e. they are qualified and registered to practise;

f. they consent to take part in the survey.

\section{Exclusion criteria}

Healthcare professionals are not eligible to participate in the survey if:

a. they are student doctors, nurses or pharmacists;

b. they work only in paediatrics (ie, do not work with adults at all);

c. they work mainly in secondary care;

$\mathrm{d}$. there is a lack of consent from the healthcare professional or they decide to quit the study at any stage and for any reason.

\section{Sample size}

The sample size is based on the estimation of the proportion of those participants who answer 'never' to the primary outcome: 'I ask patients if they have missed any doses of their medication' in each country. Using the approach in Cochran ${ }^{8}$ a sample size of 384 healthcare professionals in each country (128 people in each professional group) would enable estimation of this unknown proportion to within an absolute value of $5 \%$ with $95 \%$ confidence interval, CI.

\section{Recruitment of participants}

A mixed-method approach will be used in order to recruit participants in each country. A random sample of healthcare professionals will be sought by sampling from registers of healthcare professional bodies or associations. The number of healthcare professionals sampled and invited to participate in the survey will be based on 
reported ${ }^{9}$ response rates of healthcare professionals to online surveys of this nature. Each healthcare professional that is selected from professional registers will initially receive a letter inviting them to participate in the online survey and a project information sheet. The invitation letter will contain information about the survey as well as the web link which potential participants need in order to gain access to the survey. Reminder letters will then be sent to the healthcare professionals 3 weeks and again 5 weeks after the initial contact.

News articles to promote awareness of the survey will also be sent to healthcare professional bodies and associations for circulation through the respective organisations' websites and newsletters. The news article will also be distributed to publications whose main audience is healthcare professionals. The news article will contain information about the study as well as the web link which healthcare professionals need in order to access the survey.

All potential participants will be given the same project information and gain access to the survey via the same web link.

Data collection commenced at the beginning of July 2011. It is anticipated that data collection in all countries will cease at the end of March 2012.

\section{Questionnaire development}

There are relatively few research studies examining healthcare professional behaviour with regard to supporting patients with adherence to medication. Although it was not possible to identify any validated scales of healthcare professional behaviour in this domain, two unvalidated but published questionnaires which had been used to measure adherence behaviour among hospital-based doctors ${ }^{10}$ and cardiovascular nurses $^{11}$ were found. The ABC healthcare professional adherence questionnaire was informed by a combination of these scales, ${ }^{911}$ as well as recommendations for clinical practice from published adherence guidelines. ${ }^{2} 61213$

A subgroup of the $\mathrm{ABC}$ research team discussed, reviewed and edited potential items considered for inclusion in the questionnaire. The themes covered are:

A. perceptions of the extent of non-adherence to medication in patients;

B. beliefs about adherence to prescribed medication;

C. use of adherence enhancing interventions;

D. barriers to use of adherence enhancing interventions;

E. questions about training on adherence and use of guidelines for adherence management

\section{Description of the questionnaire}

The questionnaire is made up of 86 items in total and divided into five subsections. Below is a brief description of each subsection in the instrument.

A. Perceptions of the extent of patient non-adherence: This section contains a total of six questions split equally into two subsections. The first section asks about healthcare professionals' perceptions of nonadherence in all patients-for example: 'what percentage of all patients with a chronic condition/ illness in your country do you think do not initiate prescribed medication (ie, patients who do not take any of their prescribed medication)?' The second section asks about their perceptions of non-adherence in their own patients-for example: 'what percentage of your patients with a chronic condition/illness in your country do you think do not initiate prescribed medication (ie., patients who do not take any of their prescribed medication)?'

A five-point rating scale is provided for respondents to make their ratings, with response options of '0-15\%,' '16-35\%,' '36-65\%,' '66-85\%' and '86-100\%.'

B. Beliefs about adherence to medicines: There are seven items in this section. Participants are asked to indicate the extent to which they agree or disagree with each statement about patient adherence. For example, 'it is possible to improve patient adherence to medication.

A five-point rating scale is provided for participants to make their ratings, with options ranging from 'strongly disagree' to 'strongly agree' with intermediate labels of 'disagree,' 'neither agree nor disagree' and 'agree.' The response category 'don't know' is also available to respondents.

C. Adherence enhancing interventions used by doctors, nurses and pharmacists: This section is made up of a total of 50 questions split into five subsections. These are:

a. Assessment of adherence and its risk factors: There are eight items in this subsection. An example of an item in this section is: 'I use electronic monitoring devices to assess patients' level of adherence.' The primary outcome is included in this subsection. The wording for the question is: 'I ask patients if they have missed any doses of their medication.'

b. Providing information for carers and patients: There are nine items in this subsection. An example of an item from this section is: 'I check that patients understand the information that I have given them.'

c. Talking with patients about their medications: This subsection is made up of a total of 18 items. An example of an item from this section is: 'I ask patients what level of involvement they would like in making decisions about their treatment.'

d. Practical strategies to make medication taking easier: Eleven items make up this subsection. An example of an item from this section is: 'I help patients to tailor their medication regimen to their own lifestyle.'

e. Involving others and services to support adherence. This subsection consists of four items in total. An example of an item from this section is: 'I 
refer patients to peer mentor programmes to support medication adherence.'

The response scale for the entire section on adherence enhancing interventions is adapted from Berben $e t a l \mathrm{~s}$ survey of adherence practices by European cardiovascular nurses. ${ }^{10}$ The response options here are split into two. Respondents are first asked to indicate how often they use the intervention. A five-point rating scale is provided for participants to provide their frequency of use with responses ranging from 'never' to 'all the time' and intermediate ratings of 'occasionally,' 'sometimes' and 'frequently.' The response category 'not applicable' is made available to participants who do not use any of the interventions mentioned. Next, respondents are given the opportunity to indicate, for every intervention they use, how effective they think that intervention is. A three-point rating scale is provided; with responses ranging from 'not at all' to 'extremely,' with an intermediate category 'somewhat.' The response category 'not applicable' is provided for those who select the option 'not applicable' in column 1.

D. Barriers to the use of adherence management practices by healthcare professionals: This section contains 13 questions. An example of an item from the list is: 'I have an excessive workload that prevents me from supporting patients with medicine adherence.' A four-point rating scale is provided for participants to indicate the extent to which the items listed act as barriers to their use of adherence promoting interventions. The options range from 'not at all' to 'very much' with intermediate options of 'slightly' and 'moderately.' The response option 'not applicable' is provided for those who do not consider an item to be relevant to their work setting.

E. A final set of three questions about previous training in medication adherence and use of adherence guidelines completes the questionnaire. The questions ask whether the healthcare professional has had any training in adherence management during preregistration or postregistration training. Respondents are also asked if they make use of any practitioner guidelines to manage patient adherence. The response options are 'yes' or 'no.'

\section{Outcomes}

Primary outcome

The primary outcome is the frequency of assessing the likelihood of non-adherence: This will be based on the response to the question 'I ask patients if they have missed any doses of their medication.'

\section{Secondary outcomes}

The following secondary outcomes will be reported:

- Perception of the extent of non-adherence: This measure is based on the participants' responses to a series of six questions that ask about their perceptions of the levels of non adherence in all patients versus their patients.
- Beliefs about adherence: This is based on an assessment of healthcare professionals' beliefs about patient adherence.

- Methods used to support patients with medication taking: This is a measure of healthcare professionals' behaviour. Fifty items provide a measure of what healthcare professionals do to support patients with medicine taking.

- Barriers to the use of adherence enhancing practices: Participants are asked to indicate the extent to which the 13 items listed act as barriers that limit their use of interventions to improve adherence.

- In addition, demographic information about the respondents will be collected, including healthcare setting (eg, community setting, private or statefunded healthcare system), number of years since registration as a healthcare professional, gender, age and average length of consultations with patients about medicines.

\section{Analysis}

Analyses will be conducted by total sample, country and professional group. No interim analysis is planned.

\section{Analysis of primary outcome}

For all countries, the number and proportion of participants with the primary outcome will be reported overall and in each country, as will $95 \%$ CIs for the proportions. Comparisons between countries and professions will be performed using multilevel random models using the software MLwiN (http://www.cmm.bristol.ac.uk/ MLwiN/) for both binary and ordered categories. The software has the advantage of dealing with missing values by using all available data on each individual and not by imputing missing values.

Point estimates of the proportion of healthcare professionals who assess adherence in the entire sample (primary outcome) as well as in each country and each profession and their associated $95 \%$ CIs will be obtained.

\section{Analysis of secondary outcomes}

For the entire sample and each group, the medians, modes, IQRs and frequency distributions will be reported for each secondary outcome, and 95\% CIs for the medians will be reported. Comparisons between and within countries and professions will be performed using multilevel models using all ordered categories and which will also include demographic and other information on participants (see below). The following comparisons of outcomes will be performed:

- comparisons between the participating countries;

- comparisons between each professional group;

- comparisons by country and profession.

\section{Description of samples}

Characteristics of participants will be described for the sample as a whole, for each country and for each profession. Participants will be described in terms of their healthcare setting, type of healthcare system, 
number of years since licensed to practise, average length of time consulting with patients about medicines, age, gender and country.

\section{Data-handling}

Only the research team at Keele University will have access to the data during the study. After the study is completed, Keele University will make available the relevant data to $\mathrm{ABC}$ project partners for analysis, as appropriate.

\section{Ethics and dissemination \\ Consent}

Respondents who accept the invitation to take part in the study, and use the link provided to access the survey web page, are taken to the survey introductory page. Here, the participants are provided with information about the project, anonymity of the survey findings, an outline of what participants are required to do and how long it will take to complete the questions, an assurance that every attempt will be made to ensure the confidentiality of the data and a statement indicating that participation is voluntary and that withdrawal from the survey is possible at any stage. Potential participants are asked to click on a link to confirm that they have read the participant information before proceeding. The act of clicking on this link is considered consent to participate in the study. Access to the survey is denied unless this link is clicked.

\section{Confidentiality}

No personal information (such as names, addresses and professional licence numbers) will be collected from participants. The survey is completely anonymous, and no IP addresses will be stored or downloaded.

\section{Quality assurance}

During the preparation of the study, quality has been ensured through the process of translation and backtranslation of research questionnaires. The questionnaire and the associated survey materials have been translated into the official language(s) for each participating country. The work-flow and quality-management processes employed are certified to meet ISO 9001 Quality Management Standards. Forward translations have been performed by highly trained, approved and accredited translators who are native speakers of the target languages and fluent in English. Back-translations have been performed by persons who are native English speakers and fluent in each target language. A third individual acts as a reviewer, highlights any discrepancies between the forward and back translations, and resolves them by discussion with the translators. The respective national coordinators and their teams for each participating country also proofread each translated document and provide feedback on grammatical errors. They also provide contextual interpretation of the translations to ensure that they reflect the appropriate terminology used in each participating country. In addition to this, the online survey is piloted by at least five people in each country in order to check its technical functionality and also to check for comprehensibility and formatting errors.

The ABC research team at Keele University will have overall responsibility for the design, recruitment, management, analysis of data and interpretation of results as well as writing the report. The ABC Project partners (First Department of Family Medicine, Medical University of Lodz, Poland, Center for Health Services and Nursing Research, Katholieke Universiteit, Belgium, Aardex Group, Switzerland, Bangor University, Wales) participated in the design of the study. $A B C$ partners will manage recruitment and data collection in their respective countries and also contribute to the interpretation of the results and their dissemination. The ABC project partners share all decisions about research developments and outputs, determining decisions by vote if appropriate. The funder of the research, the FP7 programme of the European Commission, is not involved in the design or interpretation of the study.

\section{Dissemination}

The results will also be published in internal reports, in peer-reviewed scientific journals as well as via conference presentations. The results of the study will also be available to the public on the $\mathrm{ABC}$ project website (http:// www.ABCproject.eu) and via press releases in each of the participating countries.

\section{DISCUSSION}

To our knowledge, this study is the largest survey of European healthcare professionals' medication adherence perceptions, beliefs and behaviours. It is expected that the results will inform our understanding of how healthcare professionals perceive medication nonadherence. This study will also provide some insight into how healthcare professionals respond to non-adherence to prescribed medication in their patients. By gaining a deeper understanding of healthcare professionals' perceptions and behaviour with regards to non-adherence in their patients, researchers will be able to design educational interventions and training for healthcare professionals that are evidence-based and targeted at the training needs of healthcare professionals.

This study will also provide information on the interventions most frequently used by healthcare professionals and their perceptions of which interventions are most effective in managing non-adherence. It is anticipated that this will provide evidence-based knowledge of interventions which healthcare professionals have found to be effective at improving patient adherence. Healthcare professionals could use this information as a guide when making a decision about which interventions to make use of or to recommend to patients in order to improve adherence. Information on those interventions which are reported to be less effective could help to 
channel the efforts of researchers towards finding ways to improve those interventions.

The Pan-European nature of this study will provide a comprehensive data set which will enable analysis of variability observed in healthcare professionals' beliefs and behaviours across 12 European nations. It is anticipated that this knowledge of the level of variability between professions in adherence-supporting behaviour may provide a basis for promoting routine and continuous efforts to educate and modify the behaviour of healthcare professionals in order to enable them to fulfil their roles in supporting patients with medicine taking.

Author affiliations

${ }^{1}$ NPC Plus, Keele University, Keele, UK

2Division of Applied Health Sciences, School of Medicine and Dentistry, University of Aberdeen, Aberdeen, UK

${ }^{3}$ School of Computing and Mathematics, Keele University, Keele, UK

${ }^{4}$ Center for Health Services and Nursing Research, Katholieke Universiteit, Leuven, Belgium

${ }^{5}$ Sinclair School of Nursing, University of Missouri, Columbia, Missouri, USA

${ }^{6}$ School of Psychology, Bangor University, Bangor, UK

${ }^{7}$ First Department of Family Medicine, Medical University of Lodz, Lodz, Poland

Acknowledgements We thank UD Patel and $L$ Berben for their contribution to the development of the questionnaire.

Funding This study, as part of the $A B C$ project, is funded by the European Commission Seventh Framework programme (FP7 Theme Health, 2007-3.1-5, grant agreement number 223477).

Competing interests None.

Ethics approval Ethics approval was provided by the NRES Committee North West-Liverpool East (REC Reference 11/NW/0156)

Contributors The principal investigator is WC. The ABC project coordinator is PK. All authors took part in the development of the questionnaire. WC, CM, SH, SM and PJ took part in the design and development of the protocol. All authors contributed to the writing of the final manuscript. WC and CM revised the manuscript with input and advice from all authors.

Provenance and peer review Not commissioned; internally peer reviewed.

Data sharing statement No additional data available.

\section{REFERENCES}

1. Mathers C, Boerma T, Ma Fat D. The Global Burden of Disease: 2004 Update. Geneva: World Health Organization, 2008.

2. Sabaté E. Adherence to Long-Term Therapies-Evidence for Action. Geneva: World Health Organization, 2003.

3. Melnikow J, Kiefe C. Patient compliance and medical research: issues in methodology. J Gen Intern Med 1994;9:96-105.

4. Hughes DA. Economic impact of poor compliance with pharmaceuticals. Expert Rev Pharmacoecon Outcomes Res 2002;2:327-35.

5. Cleemput I, Kesteloot K, DeGeest S. A review of the literature on the economics of noncompliance. Room for methodological improvement. Health Policy 2002;59:65-94.

6. Haynes RB, Ackloo E, Sahota N, et al. Interventions for enhancing medication adherence. Cochrane Database Syst Rev 2008;(2): CD000011.

7. Dobrow MJ, Orchard MC, Golden B, et al. Response audit of an internet survey of health care providers and administrators: implications for determination of response rates. J Med Internet Res 2008;10:e30.

8. Cochran WG. Sampling Techniques. 3rd edn. New York: Wiley, 1977.

9. Braithwaite D, Emery J, de Lusignan S, et al. Using the Internet to conduct surveys of health professionals: a valid alternative? Fam Pract 2003;20:545-51.

10. Patel UD, Davis MM. Physicians' attitudes and practices regarding adherence to medical regimens by patients with chronic illness. Clin Pediatr (Phila) 2006;45:439-45.

11. Berben L, Bogert L, Leventhal ME, et al. Which interventions are used by health care professionals to enhance medication adherence in cardiovascular patients? A survey of current clinical practice. Eur $J$ Cardiovasc Nurs 2011;10:14-21.

12. Nunes V, Neilson J, O'Flynn N, et al. Clinical Guidelines and Evidence Review for Medicines Adherence: Involving Patients in Decisions About Prescribed Medicines and Supporting Adherence. London: National Collaborating Centre for Primary Care and Royal College of General Practitioners, 2009.

13. Horne R, Weinman J, Barber N, et al. Concordance, Adherence and Compliance in Medicine Taking. London: National Co-ordinating Centre for NHS Service Delivery and Organisation R \& D, 2005. 